\title{
Anthropologie des microbes
}

L'oubli de l'immunologie et la révolution du microbiome

\section{Frédéric Keck}

\section{(2) OpenEdition}

Journals

Édition électronique

URL : https://journals.openedition.org/tc/8646

DOI : $10.4000 /$ tc. 8646

ISSN : 1952-420X

Éditeur

Éditions de l'EHESS

\section{Édition imprimée}

Date de publication : 18 décembre 2017

Pagination : 230-247

ISBN : 978-2-7132-2708-0

ISSN : 0248-6016

Référence électronique

Frédéric Keck, «Anthropologie des microbes », Techniques \& Culture [En ligne], 68 | 2017, mis en ligne le 18 décembre 2019, consulté le 29 septembre 2022. URL : http://journals.openedition.org/tc/8646 :

DOI : https://doi.org/10.4000/tc.8646 


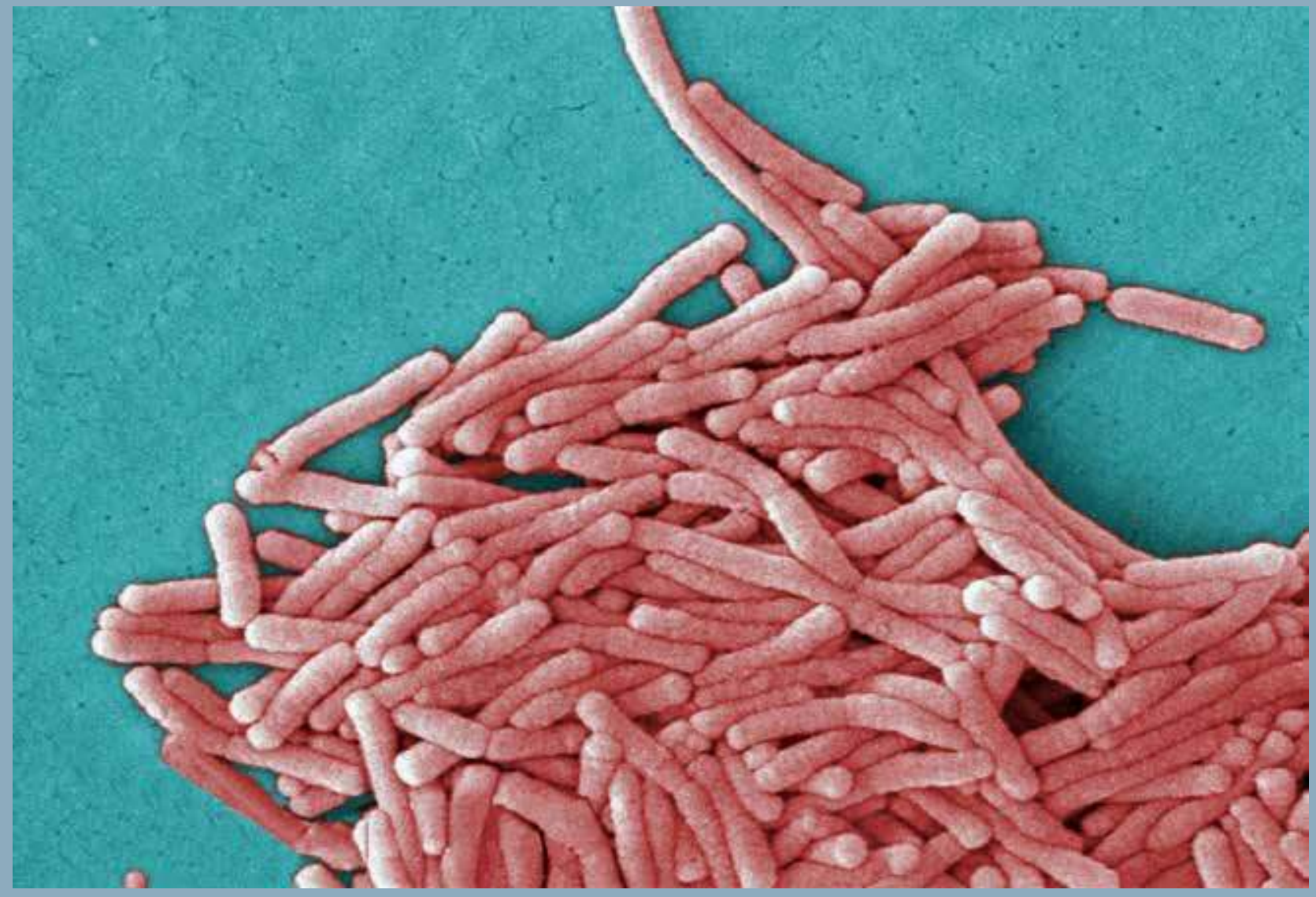




\section{Anthropologie des microbes L'oubli de l'immunologie et la révolution du microbiome}

Le programme d'une anthropologie des microbes (Benezra et al. 2012) a été inauguré par le livre de Bruno Latour, Les Microbes. Guerre et paix, paru en français en 1984, traduit en anglais en 1988 sous le titre The Pasteurization of France, et réédité en 2001 sous le titre Pasteur. Guerre et paix des microbes (précédé d'un texte théorique également rédigé en 1984, Irréductions) ${ }^{1}$. Ce livre est fondateur car il propose de considérer les microbes comme des acteurs pour une science renouvelée de la société. Il a notamment trouvé des échos aux États-Unis dans les travaux de Stefan Helmreich et Heather Paxson (Paxson 2008, Helmreich 2009, Helmreich et Paxson 2014), et en France dans ceux de Charlotte Brives (Brives 2011, Brives et Le Marcis 2015).

Je voudrais montrer dans cet article que le programme lancé par Latour, s'il a suscité de riches travaux ethnographiques sur les microbes et plus généralement sur les changements d'échelles en sciences sociales, souffre d'une double zone aveugle. L'une, volontaire, est l'immunologie, que Latour associe à la défense militaire d'un territoire, alors que cette science était pour Pasteur indissociable de la microbiologie. Lautre, involontaire, est le microbiome, un concept qui n'avait pas cours au moment où Latour écrivait son livre sur les microbes, et qui résulte d'une révolution technologique, le séquençage à haut débit, permettant de faire la carte de l'ensemble des microbes à l'intérieur d'un organisme. Cet article propose donc de prolonger le programme d'une anthropologie des microbes en intégrant ces deux domaines. 


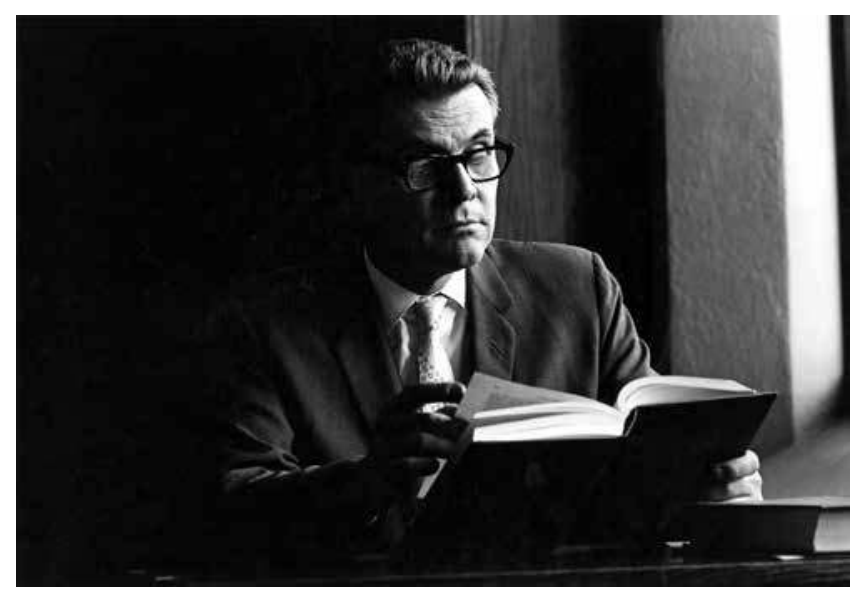

William McNeill, historien des épidémies à l'université de Chicago

\section{I'alliance entre les hommes et les microbes dans le laboratoire}

Le livre de Bruno Latour sur Louis Pasteur s'inscrit dans une double filiation anglophone qui lui permet de renverser le récit hagiographique fait en France de cette figure fondatrice ${ }^{2}$ : l'histoire de l'environnement pratiquée aux États-Unis et l'histoire des sciences pratiquée en Grande-Bretagne.

«William McNeill, 1978, Le temps des pestes. Essai sur les épidémies dans l'histoire, est l'inspirateur de ces pages. » (Latour 2001: 73, n. 1). En se rattachant à cet auteur alors peu connu en France ${ }^{3}$, Latour s'écarte volontairement d'une autre généalogie pour l'étude des transformations de la médecine: celle qui va de Georges Canguilhem à Michel Foucault, et qui articule l'expérience de la maladie aux normes de santé. De fait, l'ouvrage de William McNeill, Plagues and Peoples, est paru en 1976, la même année que le livre où Foucault lance le terme de biopolitique, La volonté de savoir. Mais alors que Foucault étudie la micropolitique du vivant comme une étape dans son analyse des formes de gouvernement et de rationalité, à mi-chemin entre la surveillance dans les prisons et les techniques de soi dans l'Antiquité, Latour s'interroge sur le rôle des microbes dans une réflexion cosmopolitique allant de l'étude de «la vie de laboratoire» avec des endocrinologues (Latour \& Woolgar 1988) à des discussions avec des géologues autour de l'Anthropocène (Latour 2015).

William McNeill (1917-2016), professeur à l'université de Chicago de 1947 à 1987, est en effet un des fondateurs de l'histoire globale et de l'histoire environnementale. Dans son grand livre The Rise of the West: A History of the Human Community publie en 1963, il s'interrogeait après d'autres (Max Weber, Oswald Spengler, Joseph Needham... ) sur les raisons pour lesquelles l'Europe a conquis le reste du monde et non l'inverse. Dans Plagues and Peoples, il développe l'hypothèse de Crosby (1972, 1986), qui sera ensuite reprise par Diamond (1997), selon laquelle les microbes furent des alliés des Européens dans leur expansion au reste du monde. Selon McNeill, les microbes ont permis à l'espèce humaine de se développer lorsqu'elle est passée des climats tropicaux de l'Afrique aux climats tempérés de l'Europe et de l'Asie. Les pestes médiévales, résultant du brassage des populations eurasiatiques du fait des grandes invasions d'Asie Centrale vers l'Europe, immunisèrent les Européens contre un certain nombre de microbes (notamment la variole) qui décimèrent les autres peuples lorsqu'ils partirent à la conquête du monde. Les Européens purent ensuite imposer aux sociétés qu'ils avaient conquises grâce aux microbes les techniques de détection et de traitement qui leur avaient permis de vivre avec ces microbes. McNeill décrit ainsi l'histoire humaine comme une coévolution entre «microparasites » et «macro-parasites» (1978: 15). 
Cette thèse fut reprise en France par Emmanuel Le Roy Ladurie, à partir de l'histoire économique de l'École des Annales. Dans un article intitulé «Un concept : l'unification microbienne du monde (XIve-XvII siècles)» et publié en 1973 dans la Revue suisse d'histoire, il rappelle que les pestes du Moyen-Âge ont tué beaucoup plus de personnes que les épidémies de l'âge moderne. La Provence, région française dont il a étudié les archives, a perdu $40 \%$ de sa population entre 1348 et 1361, dans la période où les chroniqueurs décrivent un grand nombre de cas de peste. Par contraste, les sociétés d'Amérique et d'Océanie ont été décimées par les épidémies aux XVI et XVII siècles: le Mexique central est passé de 25 millions de personnes en 1518 à 1 million en 1608. Le Roy Ladurie en conclut: «L'unification microbienne du monde, ou encore, la création, à l'échelle de l'Eurasie, puis de l'Atlantique, d'un marché commun des microbes, est passée, entre 1300 et 1600, par une phase spécialement intense, rapide, dramatique, et pourquoi ne pas le dire, apocalyptique. Les sacrifices humains, provoqués par cette globalisation des agents pathogènes, ont été pendant ces trois siècles sans commune mesure avec ce qu'ils furent ou avec ce qu'ils seront pendant les époques antérieures et postérieures. » (Le Roy Ladurie 1978:39).

Selon Le Roy Ladurie, nous serions ainsi au milieu des années 1970 dans une situation similaire à celle de l'Europe médiévale face à la peste ou de l'Amérique face à la variole: une nouvelle transformation du monde, mesurable notamment avec le réchauffement climatique, expose toute l'humanité à des pandémies à venir. De même, McNeill joue un rôle d'expert et de lanceur d'alerte dans les discussions sur les maladies infectieuses émergentes qui ont lieu aux États-Unis entre biologistes et historiens à la suite de l'éradication de la variole - qui conduit à imaginer un attentat terroriste utilisant des virus de variole conservés en laboratoire pour décimer des populations non immunisées - et à l'apparition du virus Ebola transmis des chauves-souris aux singes et aux humains en Afrique Centrale (Zylberman 2013).

En 1984 et plus encore en 2001, Latour refuse une telle position d'expert lanceur d'alerte, car il veut interroger les conditions permettant aux scientifiques de passer de l'observation des microbes en laboratoire à un diagnostic sur la société dans l'espace public. Il ne s'agit pas pour lui d'adopter une position neutre ou réflexive mais plutôt de décrire comment les acteurs sociaux passent d'une alerte à une autre - d'une attaque terroriste à une maladie infectieuse émergente au réchauffement climatique - en modifiant les connexions et les échelles. C'est ici que joue sa deuxième inspiration: l'histoire et la philosophie des sciences telle qu'elle est pratiquée en Grande-Bretagne par David Bloor, Simon Schaffer et Steven Shapin.

Il s'agit, selon le principe de symétrie préconisé par Bloor (1982) contre la tradition épistémo logique française, de décrire deux positions s'affrontant dans une controverse selon leurs arguments propres sans préjuger de leur vérité. Ainsi, Pouchet, partisan de la génération spontanée, fait la même chose que Pasteur: tous deux connectent des observations en laboratoire avec des observations hors laboratoire; mais Pasteur, équipé de son microscope et d'autres outils, arrive à convoquer un plus grand nombre d'acteurs et à construire des chaînes d'arguments plus solides. Il s'agit également, selon la méthode adoptée par Shapin et Schaffer (1993) pour décrire la discussion entre Hobbes et Boyle, de montrer que ces connexions sont aussi scientifiques que 
2. Louis Pasteur a utilisé ce microscope composé pour étudier les maladies des vers à soie entre 1868 et 1869 . Le microscope a son propre boîtier d'acajou qui contient deux lentilles et deux verres oculaires. Il est exposé à côté d'une cordelette de cocons de vers à soie. Pasteur l'a offert à la famille dont il était I'hôte durant cette période, puis le musée $\mathrm{H}$. Wellcome l'acquiert en 1926.

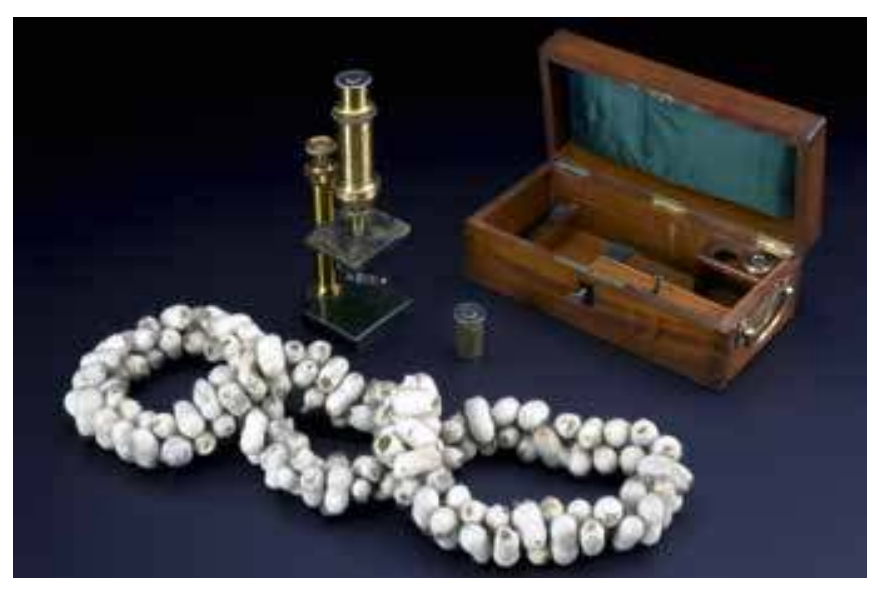
(Latour 2001: 228). politiques: c'est un modèle de société qui est en jeu dans les relations que Pasteur décrit entre les microbes, autant qu'un modèle de vérité. D'où l'attention portée aux techniques qui rendent visibles et objectives les théories scientifiques: Pasteur fait avec sa fiole à bec de cygne un travail de démonstration analogue à celui de Boyle avec sa pompe à air, produisant un modèle réduit de ce qui compose la société (Shapin \& Schaffer 1993, Latour 1997: 27).

Latour montre alors, en s'appuyant sur l'histoire de la bactériologie qui commence à s'écrire en Grande-Bretagne et en Allemagne (Enticott 2001, Worboys 2001, Mendelsohn 2002, Gradmann 2009), que cette nouvelle science des microbes a pu soutenir les Européens dans leur expansion parce qu'elle leur a permis de déplacer des procédures du laboratoire vers d'autres espaces: la campagne d'abord, où elle résout les difficultés des éleveurs et des vignerons, les colonies ensuite, où elle permet de traiter les «malades tropicales». Pasteur triomphe en vaccinant des moutons lors de l'expérience de Pouilly-le-Fort en 1882, Koch identifie le bacille responsable du choléra lors d'une expédition en Égypte en 1883, et Yersin, élève de Pasteur, découvre le bacille de la peste à Hong Kong en 1894. «S'il avait fallu faire la société coloniale avec des maîtres et des esclaves seulement, il n'y aurait jamais eu de société coloniale. Il fallait la faire avec des microbes et y ajouter le pullulement des insectes et des parasites qu'ils transportent. »

Cette proposition, qui semble traduire simplement l'histoire environnementale de William McNeill en y ajoutant les méthodes de l'histoire des sciences, est en fait une prise de position à l'intérieur de la sociologie française. Latour combine ces deux courants historiques qui se sont développés séparément pour construire une machine de guerre contre la sociologie de Pierre Bourdieu et la tradition épistémologique dans laquelle elle s'inscrit. Le point essentiel dans la démonstration de Latour est le suivant: l'opposition entre nature et société ne préexiste pas au travail de Pasteur, par exemple lorsqu'il doit connecter entre eux les microbes en laboratoire et l'État républicain, mais elle en résulte. Pasteur n’a pas découvert les microbes - ils avaient été vus pour la première fois en Hollande deux siècles plus tôt par l'inventeur du microscope, Leuweenhoek-, mais il a inventé une façon de connecter entre eux les microbes, les poules, les moutons et les vers à soie pour fabriquer une société «pastorisée». «On ne peut pas composer la société avec du social seulement. Il faut y ajouter l'action des microbes. On ne comprend rien au pastorisme si l'on ne comprend pas qu'il recompose la société différemment. » (Latour 2001: 63).

La sociologie de Pierre Bourdieu part en effet de la société comme une réalité existante qui constitue son objet propre, et la divise en catégories socioprofessionnelles ou classes sociales qui sont autant de façons de faire varier le point de vue 
sociologique. Luc Boltanski, qui accompagnera Latour dans son travail de déconstruction des classes sociales en compagnie de Laurent Thévenot et Alain Desrosières (1988), effectue ainsi dans ses premiers travaux une analyse de l'hygiène maternelle en termes de classes sociales (Boltanski 1977), au moment où des historiens montrent que les campagnes hygiéniques étaient construites par des hommes dominants pour apprendre aux femmes et aux enfants à parler «le langage des microbes» (Tomes 1998). Or Latour découvre avec le microbe pasteurien un autre principe de variation: c'est en modifiant le microbe en laboratoire que Pasteur peut l'atténuer, pour produire des vaccins qui vont intéresser les acteurs hors du laboratoire. Les oppositions entre classes sociales, entre colonisateurs et colonisés, et entre humains et animaux sont secondaires par rapport à cette potentialité que possède le vaccin de fabriquer des petites différences, constituant de proche en proche un tissu social.

«En se servant du microbe dont on peut faire varier la virulence comme d'un véritable "embrayage", [les pasteuriens] peuvent passer d'une discipline à une autre et aller d'un seul coup des contages aux phagocytes, puis de ceux-ci aux fromages et de là aussi bien aux diastases qu'aux égouts: ils vont renouveler la médecine sans jamais prendre la maladie comme objet d'étude et renouveler la politique et l'hygiène sans jamais prendre l'homme pauvre ou déchu comme unité d'analyse.» (Latour 2001 : 170). Le point essentiel ici est le suivant : ce que Latour appelle une opération de traduction - déplacement d'acteurs d'une échelle à une autre et inscription dans une chaîne narrative - ne suppose ni objet - la maladie - ni sujet - le patient. La philosophie de Michel Serres (1980) permet à Latour de décrire la communication comme un ensemble de relations de «nuisance » entre des "parasites», supprimant la notion d'hôte pour ne plus conserver que la capacité du parasite à se déplacer.

Ce déplacement soulève cependant un problème: s'il n'y a plus ni sujet ni objet, en quel sens peut-on décrire ce qui est en jeu - des microbes, des animaux, des scientifiques, l'État, la société... - comme des acteurs? Dans Les microbes: guerre et paix, Latour semble hésiter entre deux conceptions. Tantôt le microbe est décrit comme un acteur dont le sociologue peut prendre le point de vue: «La création des milieux de culture est un événement historique pour le pastorien comme pour le microbe. Je ne plaisante pas. Il y a une histoire des microbes remplie elle aussi de bruits et de fureurs. » (Latour 2001 : 134-135). Tantôt il est décrit comme un moyen d'action pour ce véritable acteur qu'est Pasteur - double du sociologue puisqu'il institue la société: «Le microbe n'est pas une idée qui flotte dans la tête des savants, c'est un moyen de locomotion pour se déplacer à travers les réseaux qu'ils veulent creuser. Le microbe est un moyen d'action, profilé pour un certain usage et un certain type de liaison et de déplacement. » (Latour 2001 : 78).

Cette hésitation est levée grâce à la théorie du Janus Bifront exposée dans La science en action. Les scientifiques multiplient les alliances avec des acteurs de toutes tailles, mais ils doivent ensuite faire comme s'ils faisaient parler les êtres qu'ils se contentent de connecter. Ils attribuent alors à une nature inerte et objective ce qui doit être plutôt décrit comme des hybrides de natureculture - si l'on définit la culture par l'inventivité humaine. Ce sont donc bien les microbes qui agissent, mais c'est Pasteur qui est responsable de leur action: «Pour recruter des alliés il faut 


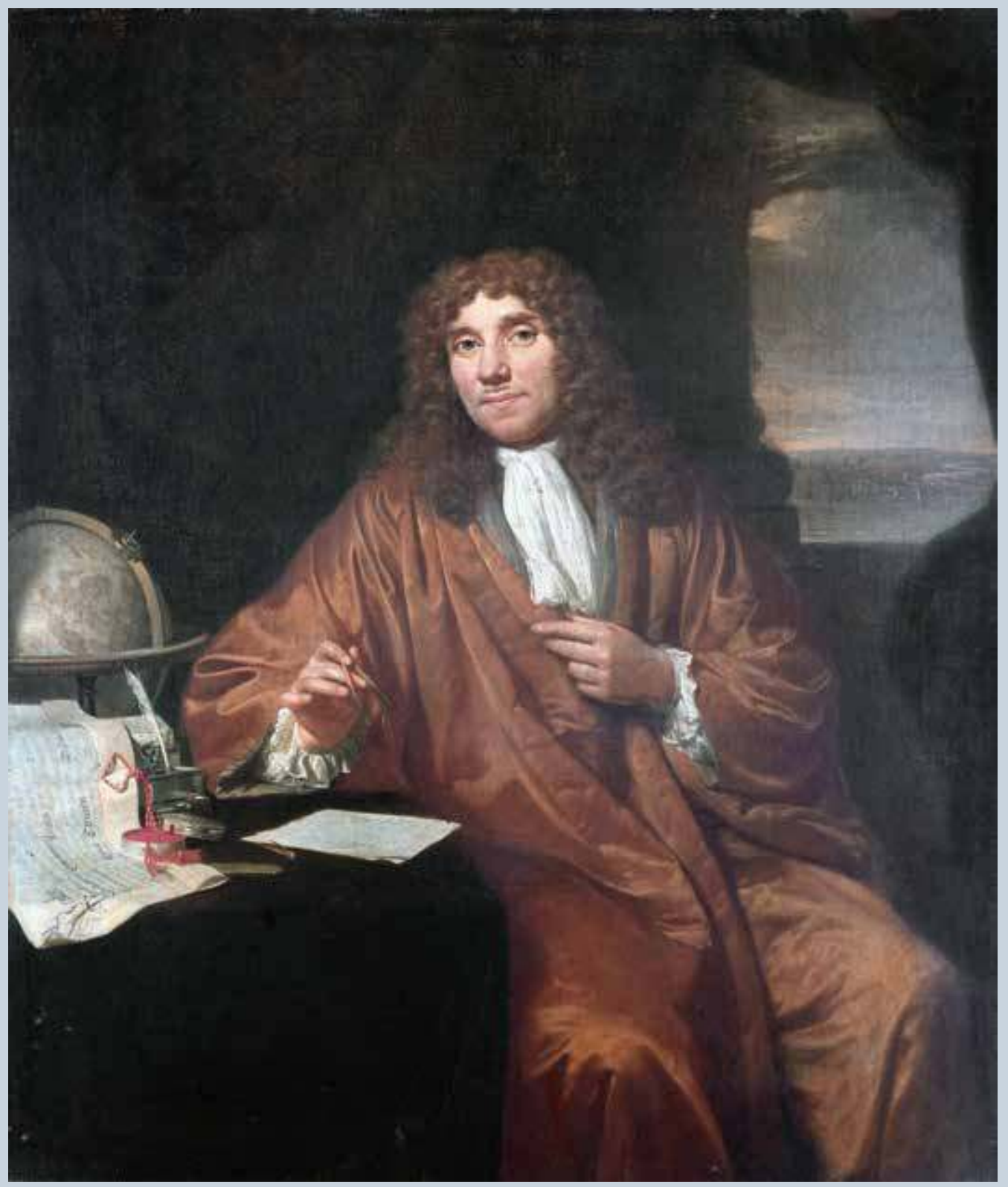

Anthonie van Leeuwenhoek (1632-1723), by Jan Verkolje (I)

Anthonie van Leeuwenhoek, inventeur du microscope, est ici représenté comme l'explorateur de nouveaux mondes avec un globe et un compas, selon les conventions de la peinture flamande. Il écrivit de nombreuses lettres à la Royal Society de Londres, dont l'une dans laquelle il affirme de façon célèbre qu'il a vu plus d'êtres vivants dans sa propre bouche que le roi d'Angleterre ne peut en rêver dans son royaume. 


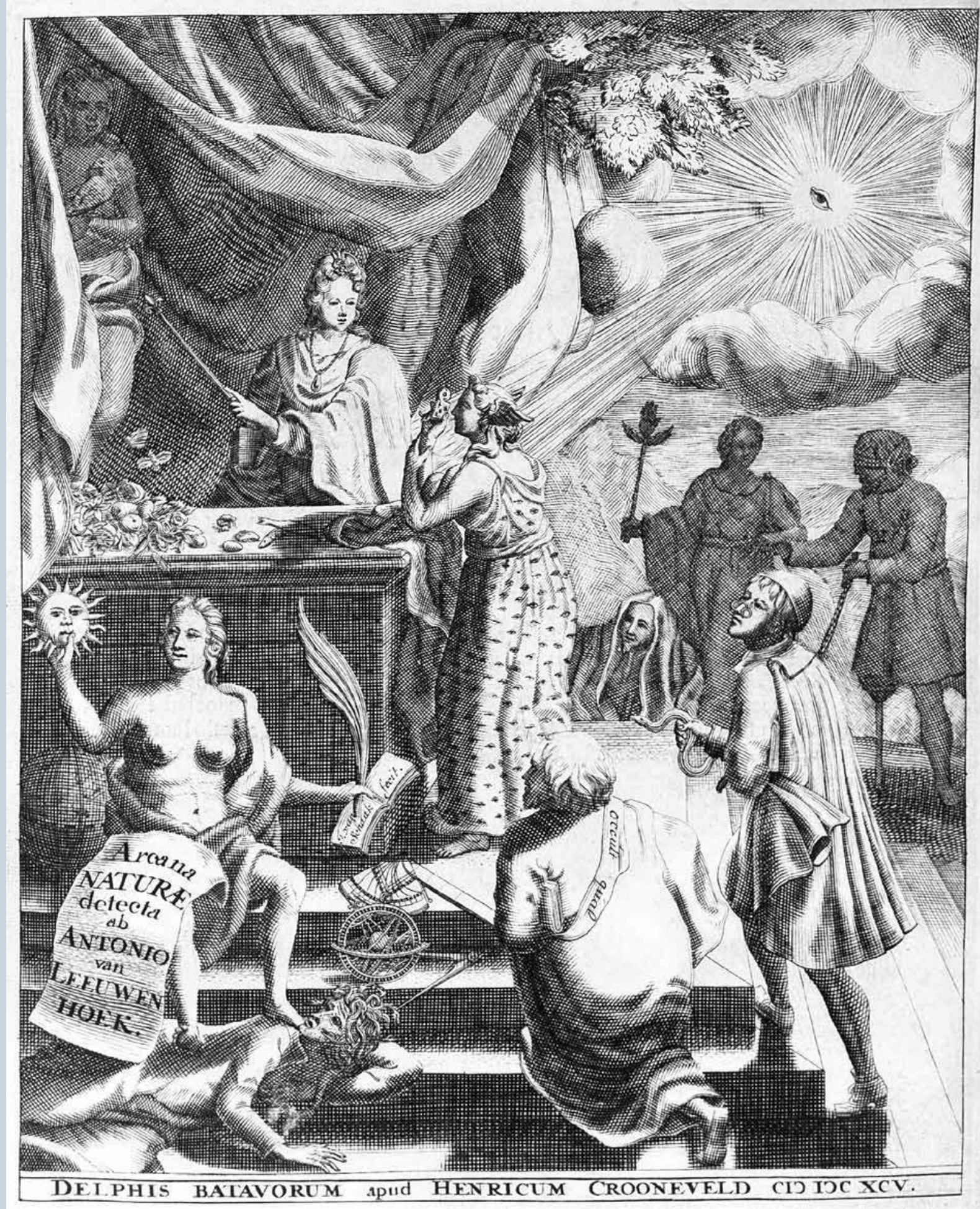


que vous alliez aussi loin que possible, et fassiez autant de compromis que nécessaire, alors que pour vous attribuer la responsabilité, il convient au contraire de limiter au maximum le nombre des acteurs. » (Latour 1995: 287). Latour résout ainsi les paradoxes de la modernité. Les modernes présentent comme des contradictions insolubles ce qui n'est qu'une différence de points de vue sur une chaîne d'actions qu'opèrent toutes les sociétés. La contradiction apparente entre le microbe et la société - entre le sale et le propre, entre le petit et le grand, entre la nature et la culture - n'est qu'une différence de taille entre des acteurs connectés par Pasteur.

Pourquoi alors la société se définit-elle par opposition avec le microbe? Comment expliquer que les modernes soient si attachés au social comme ce qui leur est propre et qui les grandit? Pour répondre à cette question il faut affronter les paradoxes de l'immunité.

\section{Les normes de l'immunité et la définition du soi}

En décrivant Pasteur comme un médiateur, Latour prend au sérieux son rôle d'entrepreneur industriel, qui fut au principe de la construction de l'Institut Pasteur comme partenariat publicprivé. Mais il laisse de côté son ambition théorique, qui était pourtant au cœur de son engagement scientifique dans la cristallographie à l'École normale supérieure. Pasteur était en effet fasciné par une question venue de la chimie: comment expliquer les propriétés de la matière qui engendrent la vie? Il avait trouvé dans l'asymétrie des cristaux une telle propriété, et en avait tiré toutes les conséquences pour l'étude des microbes. Pasteur peut en effet être décrit comme celui qui a théorisé ce qui restait une simple découverte empirique lorsque Edward Jenner rendit public au début du dix-neuvième siècle le principe de la vaccination développé depuis longtemps par les éleveurs anglais: un microbe atténué peut protéger un organisme contre d'autres microbes analogues par la trace qu'il y laisse. C'est le fondement de la théorie de l'immunité. Paradoxalement, alors que Latour a appliqué à Pasteur un principe de symétrie en histoire des sciences, c'est la découverte du rôle ontologique de l'asymétrie dans les relations entre les vivants qui fait la véritable innovation de Pasteur.

Anne-Marie Moulin a décrit la rencontre entre Louis Pasteur et Élie Metchnikoff comme l'avènement de cette théorie de l'immunité qui manquait à la microbiologie. Metchnikoff est un zoologiste russe qui applique la théorie darwinienne en décrivant non pas une lutte à l'intérieur d'une espèce pour la survie par sélection, mais une lutte entre espèces par la digestion. Selon Metchnikoff, grand observateur des oursins et des étoiles de mer, les macro-organismes entrent en relation les uns avec les autres par la façon dont leurs micro-organismes s'entre-dévorent. C'est le phénomène de la phagocytose, que Metchnikoff pose au fondement de l'immunité: l'organisme se défend contre des menaces extérieures par des cellules qui avalent et digèrent les microbes agresseurs (Metchnikoff 1901).

Latour voit dans cette théorie de l'immunité apportée par Metchnikoff à l'Institut Pasteur une «trahison» de l'invention pasteurienne, puisqu'elle conduit selon lui à faire des microbes 
« les cas particuliers d'un problème général: l'intégrité de l'organisme» (Latour 2001 : 174). Latour est ici dépendant d'une critique de l'immunologie comme idéologie sécuritaire, partagée par de nombreux analystes au cours des trente dernières années, (Haraway 1991, Cohen 2009, Esposito 2010), et il lui oppose les alliances diplomatiques de la microbiologie. Mais comme le remarque Anne-Marie Moulin dans son histoire de l'immunologie, Latour privilégie ainsi le point de vue pratique de l'entrepreneur libéral contre le point de vue théorique du penseur républicain.

«La médecine pastorienne ne reposait pas sur des hypothèses théoriques touchant l'immunité mais sur un programme empirique d'immunisation faisant appel à l'atténuation des micro-organismes. La première génération des pastoriens adhère à un transformisme discret de type lamarckien: la modification se fait grâce à la manipulation de l'environnement de la bactérie, tour de passe-passe qui transfère les méthodes fumeuses des hygiénistes du vaste monde où elles étaient aléatoires au petit monde clos du laboratoire. Bruno Latour a fait de ce changement d'échelle une des conditions de la réussite du pastorisme. Appareillage et maniabilité résultent de la miniaturisation.» (Moulin 1991: 47).

Comment l'immunologie rend-elle possible ce changement d'échelle dont Latour fait la condition pratique du succès de la médecine pasteurienne? Dans le contexte de la rivalité entre la France et l'Allemagne, Pasteur cherchait à penser avec les microbes les relations entre ce grand organisme qu'est l'État et ces micro-organismes que sont les individus. Metchnikoff lui apporte, avec l'évolutionnisme darwinien revu par la zoologie russe, la solution à ce problème. Un microbe qui agresse un organisme est analogue à un individu qui fait défaillir un État: ce phénomène incompréhensible du point de vue bio- logique devient entièrement clair du point de vue immunologique, puisque ce sont toujours des microbes qui agressent des microbes. La guerre biologique doit donc être décrite selon Pasteur à chaque niveau ontologique où elle se produit si l'on veut en comprendre les effets. «La théorie de Metchnikoff avait en outre pour son esprit ce côté satisfaisant qu'elle égalisait les forces en présence. Il y a quelque chose de disproportionné dans une bactérie qui tue un bœuf. On comprend mieux une lutte localisée entre les leucocytes du bœuf et les microbes envahisseurs qui périssent s'ils sont trop affaiblis ou trop peu nombreux.» (Duclaux 1896: 391, cité in Moulin 1991 : 52).

Vu sous cet angle, il est frappant de constater à quel point la biologie de Pasteur, complétée par la théorie de Metchnikoff, est proche de la sociologie de Durkheim. Si Latour introduit les microbes comme de nouveaux acteurs en sociologie, il reste que Durkheim a introduit le problème de l'immunité en sociologie. Latour dira vingt ans après son livre sur Pasteur qu'il lui manquait de connaître la sociologie de Tarde pour expliquer à son public français ce qu'il

\section{Élie Metchnikoff}

Après avoir travaillé en Russie et en Allemagne, Élie Metchnikoff rejoignit l'Institut Pasteur, où il développa ses idées sur l'immunité et la phagocytose. 


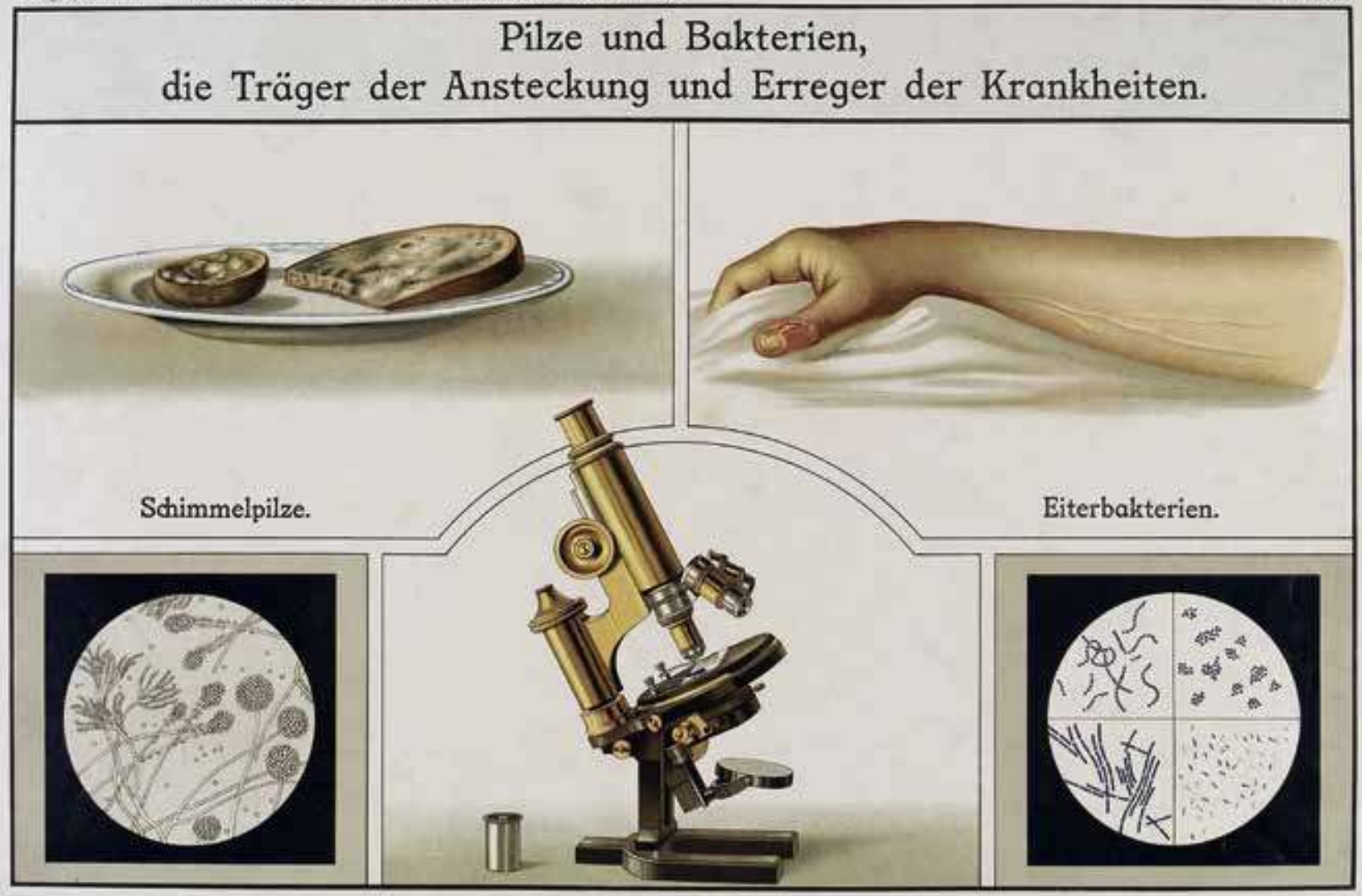

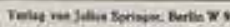

\section{Dangers pour la santé des enfants en bas âge: microbes et bactéries}

Les microbes dans leur état naturel (sur du pain moisi et sur un pouce suppurant) et les microbes comme vus par le microscope, au centre. Lithographie en couleur de l'Atlas der Hygiene des Säuglings und Kleinkindes (Atlas de I'hygiène du nourrisson et du petit enfant) $n^{\circ} 25$ by Leopold Langstein et Frits Rott, Berlin: Julius Springer, 1922. 


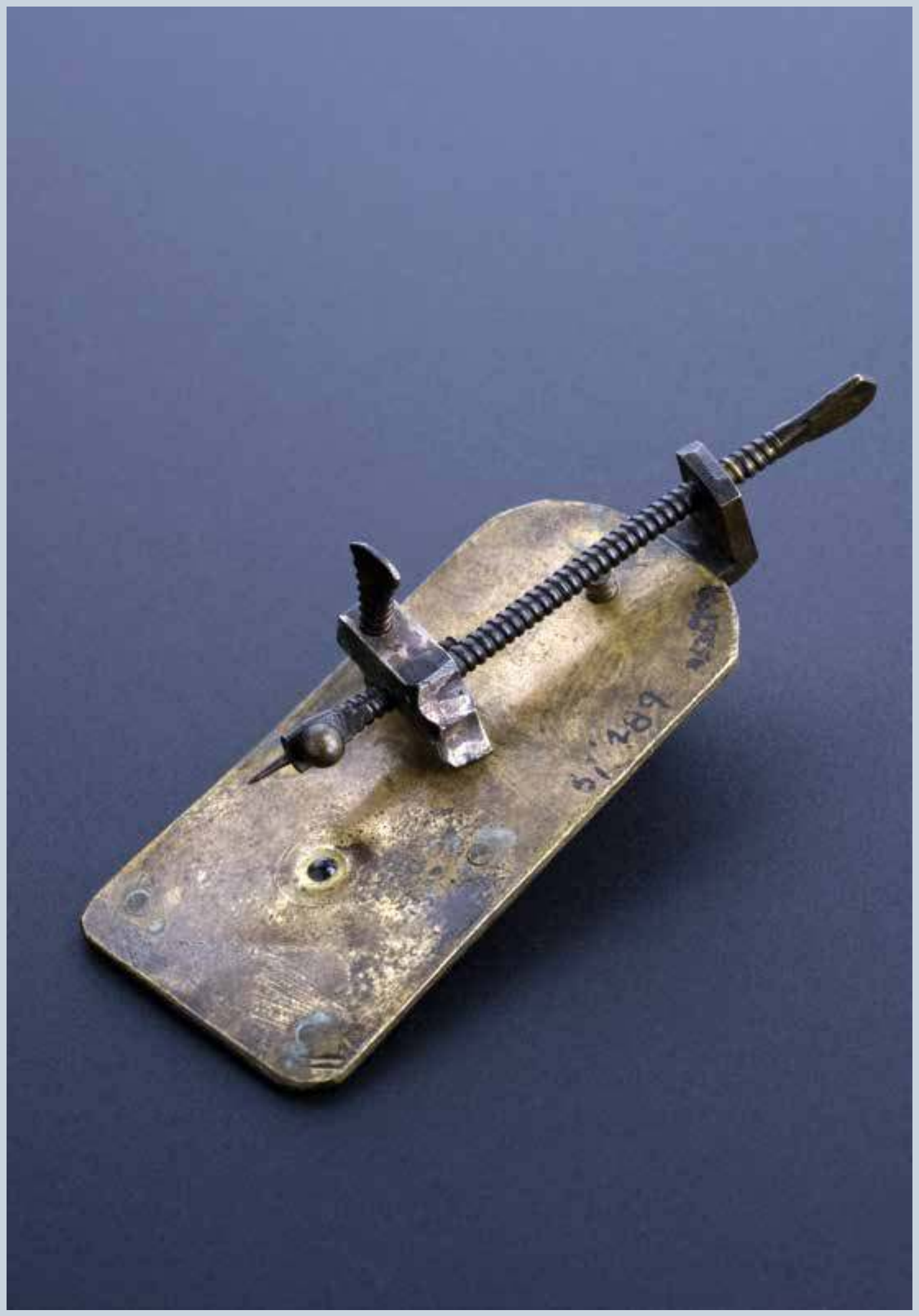


faisait (Latour et Lépinay 2006). Cependant Tarde ne parle jamais de microbes mais de contagion. Comme l'a récemment démontré Paul-André Rosenthal (2011), le problème de Tarde est de décrire comment s'arrêtent les flux contagieux de désirs et de croyances qui constituent les relations sociales. Si Tarde ne pose pas plus le problème du microbe qu'il ne pose celui de l'individu, ce problème est central chez Durkheim qui part du même phénomène que Tarde, le caractère contagieux du crime, mais en lui donnant une solution différente: l'État répond à une agression en catégorisant les individus, c'est-à-dire en les soumettant à une sanction dans l'espace du tribunal où ils deviennent visibles.

Dans le chapitre des Règles de la méthode sociologique consacré aux rapports entre le normal et le pathologique, Durkheim montre en effet que le crime est «normal» dans une société, puisqu'il dépend de la façon dont celle-ci définit ce qui relève de son ordre et ce qui doit rester extérieur. À l'inverse, explique Durkheim, la vie sociale peut apparaître pathologique aux individus, puisqu'elle consiste essentiellement en sanctions qui leur imposent une souffrance. La vie sociale peut ainsi être comprise comme une maladie utile, sur le modèle du vaccin contre la variole qui protège l'organisme en administrant un microbe atténué. «La maladie ne nous laisse pas toujours désemparés, dans un état de désadaptation irrémédiable; elle nous contraint seulement à nous adapter autrement que la plupart de nos semblables. Qui nous dit même qu'il n'existe pas de maladies qui, finalement, se trouvent être utiles? La variole que nous nous inoculons par le vaccin est une véritable maladie que nous nous donnons volontairement, et pourtant elle accroît nos chances de survie. Il y a peut-être bien d'autres cas où le trouble causé par la maladie est insignifiant à côté des immunités qu'elle confère. » (Durkheim 2010: 161).

Ce passage montre les analogies entre la pensée de Durkheim et celle de Pasteur, qui relèvent de la même théorie républicaine en cours de formation. Durkheim est parti du problème de l'inégalité comme caractéristique de la vie sociale (par différence avec la vie biologique) pour découvrir dans la sanction (répressive ou restitutive) une façon de corriger, par le rapport normé entre État et individu, ces inégalités. Pasteur est parti du problème de l'asymétrie comme caractéristique de la vie biologique (par différence avec la matérialité chimique) pour découvrir dans le rapport immunitaire entre l'organisme et les microbes une façon de réguler ces asymétries. Latour, partant du problème de l'innovation comme caractéristique de la vie en général (dont il ne distingue pas les niveaux même s'il en décrit les échelles ${ }^{4}$ ), laisse de côté la norme et l'immunité.

Pourtant les anthropologues dont il se réclame partent précisément du problème durkheimien de la norme. Marc Augé, dont les travaux sur la sorcellerie africaine permettent à Latour de penser les chaînes d'imputation (Augé 1982, cité in Latour 1997), se situe dans le cadre de l'anthropologie politique de Georges Balandier, attentive aux situations dans lesquelles les normes se reformulent. Philippe Descola, dont les travaux sur les Achuar d'Amazonie sont cités par Latour avec admiration, se situe dans le cadre de l'anthropologie structuraliste de Claude Lévi-Strauss et Maurice Godelier, pour laquelle la pensée agit sur l'environnement par la façon dont elle le norme et l'organise, comme le montre ce passage cité par Latour: "L'homéostasie des "sociétés froides" de l'Amazonie résulterait alors moins du refus implicite de l'aliénation politique dont Clastres créditait les sauvages que de l'effet d'inertie d'un système de pensée qui 
ne peut se représenter le procès de socialisation qu'à travers les catégories normant le fonctionnement de la société réelle. » (Descola 1986: 405, cité in Latour 1997: 62).

Que le problème de la norme et du niveau d'existence, par distinction avec le problème du nombre et de l'échelle des réseaux, soit au cœur de la microbiologie et de son complément nécessaire qu'est l'immunologie, c'est ce que montre le programme de recherche contemporain autour du microbiome.

\section{La révolution du microbiome}

Depuis une dizaine d'années, les possibilités offertes par le séquençage génétique à haut débit révèlent le rôle des microbes commensaux, qui vivent en symbiose avec l'organisme humain. En cartographiant la diversité de ces microbes qui représentent la plus grande partie de l'information génétique contenue dans l'organisme (l'ADN humain censé définir l'identité n'en étant qu'une partie réduite), cette révolution technologique a permis de mieux décrire le rôle des microbes commensaux dans la régulation de l'organisme. On parle de microbiome (sur le modèle de la notion de génome) pour qualifier l'ensemble des microbes contenus dans un organisme dont le séquençage permettrait de comprendre les variations (Turnbaugh et al. 2007, Cho \& Blaser 2012, Gilbert et al. 2012, McFallNgai et al. 2013, Lorimer 2016, Young 2017). On a notamment pu supposer que, face à un pathogène agressif ou à un stress environnemental, la flore microbienne se transformerait rapidement pour «digérer» le choc. Les microbes commensaux ont en effet une capacité d'adaptation infiniment plus rapide que les organismes qui les hébergent, car ils se répliquent par transfert horizontal de gènes et non, comme les cellules humaines, par descendance verticale.

Ce nouveau paradigme, qui transforme l'ensemble des sciences du vivant, vient reformuler un problème central de l'immunologie. À la suite des travaux de Metchnikoff, qui proposait une approche physiologique et cellulariste de l'immunité comprise comme digestion et valorisait déjà le rôle de certaines bactéries probiotiques contenues dans les yaourts, l'école allemande menée par Karl Lansteiner avait privilégié une approche chimique et humorale de l'immunité comprise comme inflammation, notamment à partir d'une classification des groupes sanguins permettant d'expliquer les affinités et les rejets dans la transfusion. Une synthèse de ces deux approches fut proposée par l'Australien Frank Macfarlane Burnet au milieu du vingtième siècle, définissant l'immunité par la tolérance pour le soi et le rejet du non-soi. Mais le concept de «soi » (self) restait confus chez Burnet, et à la limite idéologique. Il restait alors à décrire comment l'organisme reconnaît par apprentissage ce qui est soi et ce qui est non-soi, donc distinguer l'immunité innée (chez tous les organismes) et l'immunité acquise (chez les organismes vertébrés et chez certains invertébrés). Plutôt que de supposer un soi constitué comme une mémoire que l'organisme garde de ses agressions par des agents extérieurs, il fallait décrire comment les couples antigène-anticorps (qui permettent la reconnaissance de l'information d'un microbe 
invasif par une cellule immunitaire) se forment et se transforment dans l'histoire de l'organisme à l'occasion de rencontres avec des agents extérieurs (Moulin 1991, Pradeu 2012).

Or c'est précisément ce que rendent concevable les découvertes récentes sur le microbiome. Mettant fin à l'idéologie du soi immunitaire (selon laquelle l'organisme aurait un système de défense organisé autour d'organes centraux comme le thymus et la moelle osseuse [bone marrow], producteurs des lymphocytes $\mathrm{T}$ et $\mathrm{B}$ ), elles suggèrent que les microbes commensaux communiquent entre eux par une cascade de signaux de façon à réguler l'inflammation qui répond à un microbe invasif. Les cellules immunitaires doivent être comprises moins comme un système de douanes et de barrages interdisant le passage d'un microbe étranger que comme un «moteur de recherche» permettant de faire correspondre l'antigène de ce microbe à un récepteur cellulaire présent dans l'organisme (Napier 2012, Kourlisky 2014). Le problème de l'immunité est donc un problème de régulation de l'information: il s'agit de trouver dans l'organisme une information compatible avec celle que porte le microbe invasif, de façon à lui permettre de se développer dans cet organisme considéré comme un nouvel «hôte» ou de le rejeter.

Il y a ainsi dans la relation entre le microbe et la cellule-hôte un type de relation qui pourrait servir de modèle pour décrire toutes les autres relations sociales. Le problème, saisi immunologiquement, est en effet de trouver la forme adéquate permettant au microbe de s'attacher à la cellule-hôte sans être rejeté par elle (problème qualifié en immunologie de «tolérance», même s'il n'a rien de moral). Or la notion de forme manque dans la sociologie de Latour parce qu'il a rejeté l'héritage structuraliste des sciences humaines. Il utilise la notion d'inscription, en lien avec l'anthropologie de l'écriture développée par Jack Goody (Latour 1997), contre l'analyse de la «pensée sauvage » par Lévi-Strauss, et la sémiotique de Greimas (et Courtès 1979), qui définit les êtres comme des acteurs dans des chaînes narratives, contre la sémiotique de Saussure. Pourtant la sémiotique de Saussure et Lévi-Strauss est plus à même de décrire les relations entre antigène et anticorps - sur le modèle des relations entre signifiant et signifié - que celle de Greimas et Goody. Il faut en effet prendre au sérieux le fait que l'immunologie est un langage, et que ce langage s'écrit à travers l'information génétique (Moulin 1991). On ne comprend pas ce que fait un microbe si on le décrit comme un acteur - et Latour lui-même hésite à aller jusque-là - mais on comprend qu'un morceau d'information devienne compatible avec un autre morceau d'information si on pense cet accommodement sur le modèle des relations entre les signes linguistiques. Loin que le langage serve de métaphore pour construire l'immunologie comme théorie des relations entre le soi et l'autre, l'immunologie permet de décrire réellement comment les signes fonctionnent au niveau biologique, c'est-à-dire comment la forme d'un vivant produit une norme sociale par la façon dont elle communique avec une autre forme.

Eduardo Kohn a récemment proposé d'articuler le concept de signe avec celui de forme pour décrire la vie des Runa, un groupe vivant dans la forêt d'Amazonie. Il montre que les humains communiquent avec des non-humains par des signes, notamment par le rêve qui précède la chasse, et sont contraints par les formes de leur environnement. Il écrit : «Étant donné les défis posés par la nécessité d'apprendre à vivre avec une variété toujours plus grande de formes de vie - qu'il s'agisse d'animaux de compagnie, de mauvaises herbes, d'animaux nuisibles, de 
commensaux, de nouveaux pathogènes, d'animaux "sauvages" ou de "mutants" technoscientifiques-, il est non seulement crucial mais aussi urgent de développer une manière précise d'analyser dans quelle mesure l'humain est tout à la fois distinct de, et en continuité avec, ce qui se trouve au-delà de lui. » (Kohn 2017: 30). Kohn reprend ainsi un enseignement de Lévi-Strauss dans son «Apologue des amibes» (2000) : la façon dont les microbes communiquent entre eux par des signaux est analogue à la façon dont les chasseurs communiquent avec les non-humains. C'est ce que confirme l'immunologie, en tant qu'elle est une science des relations de prédation et de digestion entre les microbes: les vivants ne cessent de se traquer, de se leurrer, de s'éviter, et l'ensemble de ces stratégies leur permet de communiquer et de transformer l'information qui les constitue. Plutôt que dans une histoire du "pastoralisme», il faudrait donc inscrire Pasteur dans une histoire des «chasseurs de microbes» (De Kruif 1926, Perrey 2012).

La révolution du microbiome permet ainsi de transformer l'appareil conceptuel et sémiotique autour duquel s'est constituée l'anthropologie des microbes à la suite de l'ouvrage fondateur de Bruno Latour: considérer les microbes comme des populations communiquant entre elles par des signaux plutôt que comme des acteurs inscrits dans un réseau, partir des asymétries et des discontinuités entre les êtres plutôt que des symétries et des continuités, dégager des niveaux d'existence plutôt que des échelles de grossissement.

\section{Notes}

1. Le changement de titre et le complément théorique en 2001 accentue et ouvre véritablement le programme général d'une anthropologie des microbes, alors que l'ouvrage de 1984 est davantage une sociologie des sciences écrite sur le modèle de Tolstoï. Mais le texte de l'ouvrage et la démonstration restent inchangés.

2. Sur le véritable culte laïque rendu à Pasteur, cf. Perrey (2005). La tradition des biographies consacrées à Pasteur va de Duclaux (1896) à Dagognet (1967) en passant par Dubos (1955). Elle a été renouvelée à l'occasion du centenaire de la mort de Pasteur: cf. Latour 1994.
3. L'idée selon laquelle de nouveaux microbes pathogènes émergent des transformations des relations entre les hommes et leur environnement avait cependant déjà été formulée par le médecin pasteurien Charles Nicolle (1930).

4. Alors que l'échelle grossit un être en changeant de point de vue, obligeant ainsi à changer d'échelle pour construire du collectif, un niveau organise des activités selon des modalités conflictuelles qui obligent à passer à un autre niveau pour atténuer ces conflits.

\section{I'auteur}

Après une thèse sur l'histoire de l'anthropologie dans ses rapports avec la philosophie, Frédéric Keck a mené des recherches sur la grippe aviaire dans le cadre du Laboratoire d'anthropologie sociale au CNRS, et dirige actuellement le département de la recherche et de l'enseignement du musée du quai Branly. 


\section{Iconographie}

Image d'ouverture. Légionnelle. Vue colorisée d'un microscope électronique à balayage (MEB) avec grossissement modéré à $8000 \mathrm{X}$, représentant un important groupe de bactéries Legionella pneumophila à Gramnégatif. (C) Janice Haney Carr.
1. Avec l'aimable autorisation du Special Collections Research Center.

2. 4. 6. \& 7. (C) Wellcome Images.

3. Anthonie van Leeuwenhoek (1632-1723), par Jan Verkolje (I), Rijksmuseum, Amsterdam.

5. Portrait d'Élie Metchnikoff par Nadar.

\section{Remerciements}

L'auteur remercie Anne-Marie Moulin et Sophie Houdart pour leurs commentaires sur ce texte.

\section{Références}

Augé, M. 1982 Génie du paganisme. Paris: Gallimard. Benezra,A., DeStefano J. \& J.I. Gordon 2012 «Anthropology of microbes», PNAS, doi:10.1073/pnas.1200515109.

Bloor, D. 1982 Sociologie de la logique ou les limites de l'épistémologie. Paris: Pandore.

Boltanski, L. 1977 Prime éducation et morale de classe. Paris: Mouton.

Brives, C. 2011 « La levure amie des hommes. Les organismes expérimentaux dans la production du savoir scientifique» in S. Houdart, O. Thiery (dir.) Humains non humains. Comment repeupler les sciences sociales. Paris: La Découverte: 199-207.

Brives, C.\& F. Le Marcis 2015 « Réimaginer des communautés? Le traitement précoce contre le VIH/sida en Côte d'Ivoire», Terrain 64: 84-103.

Cho, I. \& M. J. Blaser 2012 «The human microbiome: At the interface of health and disease», Nature Reviews 13: $260-270$

Cohen, E. 2009 A Body Worth Defending: Immunity, Biopolitics, and the Apotheosis of the Modern Body. Cornell: Duke University Press.

Crosby, A. 1972 The Columbian Exchange: Biological and Cultural Consequences of 1492. Wesport, Connecticut / Londres: Greenwood Publishing Group.

- 1986 Ecological Imperialism: The Biological Expansion of Europe, 900-1900. Cambridge: Cambridge University Press.

Dagognet, F. 1994 [1967] Pasteur sans la légende. [Méthodes et doctrines dans l'ouvre de Pasteur. Paris: Puf] rééd. Paris: Les Empêcheurs de penser en rond.

De Kruif, P. 1926 Microbe Hunters. New York: Harcourt-Brace.

Descola, P. 1986 La nature domestique. Symbolisme et praxis dans l'écologie des Achuar. Paris: Éditions de la MSH.
Desrosières, A. \& L. Thévenot 1988 Les catégories socioprofessionnelles. Paris: La Découverte.

Diamond, J. 1997 Guns, Germs, and Steel: The Fates of Human Societies. New York: Norton\&Company's. Traduit de l'anglais par P. E. Dauzat: 2000 De l'inégalité parmi les sociétés. Essai surl'homme et l'environnement dans l'histoire. Paris: Gallimard.

Duclaux, E. 1896 Pasteur. Histoire d'un esprit. Paris: Masson.

Dubos, R. 1995 [1955] Louis Pasteur: Franc-tireur de la science. [Paris: Puf], rééd. (préface de B. Latour). Paris: La Découverte.

Durkheim, E. 2010 [1895] Les règles de la méthode sociologique. Paris: Flammarion

Eberl, G. 2010 «A New Vision of Immunity: Homeostasis of the Superorganism», Nature 3(5): 450-460

Enticott, G. 2001 «Calculating nature: The case of badgers, bovine tuberculosis and cattle», Journal of Rural Studies 17(2): 149-164.

Esposito, R. 2010 Communauté, immunité, biopolitique. Traduit de l'italien par B. Chamayou. Paris: Les prairies ordinaires.

Gilbert, S. F., Sapp, J. \& A. I. Tauber 2012 «A Symbiotic View of Life: We Have Never Been Individuals», The quarterly review of biology 87(4): 325-341.

Gradmann, C. 2009 Laboratory Disease: Robert Koch's Medical Bacteriology. Baltimore: Johns Hopkins University Press.

Greimas, A.J. \& J. Courtès 1979 Sémiotique. Dictionnaire raisonné de théorie du langage. Paris: Hachette.

Haraway, D. 1991 «The biopolitics of postmodern bodies: Constitutions of self in immune system discourse » in Simians, Cyborgs, and Women: The Reinvention of Nature. New York: Routledge: 203-230. 
Helmreich, S. 2009 Alien Ocean: Anthropological Voyages in Microbial Seas. Berkeley: University of California Press.

- 2014 «Homo Microbis: The Human Microbiome, Figural, Literal, Political» Threshold 42 : 52-59.

Helmreich, S. \& H. Paxson 2014 « Perils and promises of microbial abundance: Novel natures and model ecosystems, from artisanal cheese to alien seas », Social Studies of Science 44(2): 165-193.

Kohn, E. 2017 Comment pensent les forêts. Vers une anthropologie au-delà de l'humain. Traduit de l'anglais par G. Delaplace. Bruxelles: Zones sensibles.

Kourilsky, P. 2014. Le jeu du hasard et de la complexité. La nouvelle science de l'immunologie. Paris: Odile Jacob.

Latour, B. 1994 Pasteur. Une science, un style, un siècle. Paris: Librairie Académique Perrin-Institut Pasteur.

— 1995 La science en action. Paris: La Découverte.

—1997 Nous n'avonsjamaisété modernes. Essai d'anthropologie symétrique. Paris: La Découverte.

- 2001 Pasteur. Guerre et paix des microbes suivi de Irréductions. Paris: La Découverte.

—2015 Face à Gaïa. Huit conférences sur le Nouveau Régime Climatique. Paris: Les Empêcheurs de penser en rond / La Découverte.

Latour, B. \& V. Lépinay 2006 Introduction à Gabriel Tarde, in Psychologie économique. Paris: Les Empêcheurs.

Latour, B. \& S. Woolgar 1988 La vie de laboratoire. Paris: La Découverte.

Le Roy Ladurie, E. 1978 «Un concept: l'unification microbienne du monde (XIve-XviI ${ }^{e}$ siècles)», Le territoire de l'historien, vol.2. Paris: Gallimard: 37-97.

Lévi-Strauss, C. 2000 «Apologue des amibes» in J.-L. Jomard, E. Terray, M. Xanthakou (dir.) En substances. Textes pour Françoise Héritier. Paris: Fayard: 493-496.

Lorimer, J. 2016 « Gut buddies, multispecies studies and the microbiome», Environmental Humanities 8(1): 57-76.

McFall-Ngai, M. et al. 2013 «Animals in a bacterial world, a new imperative for the life sciences», PNAS 110(9): 3229-3236.

McNeill, W. 1963 The Rise of the West: A History of the Human Community. Chicago: University of Chicago Press.

— 1978 [1976] Plagues and Peoples. New York: Anchor Press. Traduit de l'américain Le temps des pestes. Essai sur les épidémies dans l'histoire, Paris, Hachette.
Mendelsohn, A.J. 2002 «"Like All That Lives": Biology, medicine and bacteria in the age of Pasteur and Koch", History and Philosophy of the Life Sciences 24: 3-36.

Metchnikoff, E. 1901 L'immunité dans les maladies infectieuses. Paris: Masson.

Moulin, A.-M. 1991 Le dernier langage de la médecine. Histoire de l'Immunologie, de Pasteur au SIDA. Paris: Puf.

Napier, D. 2012 «Nonself help: How immunology might reframe the enlightenment », Cultural Anthropology 27 (1): 122-37.

Nicolle, C. 1930 Naissance, vie et mort des maladies infectieuses. Paris: Alcan.

Paxson, H. 2008 « Post-pasteurian cultures: The microbiopolitics of raw-milk cheese in the United States», Cultural Anthropology 23(1): 15-47.

Perrey, C. 2005 «Les figures du sacré à l'Institut Pasteur», L'Homme 3(175-176): 345-368

Pradeu, T. 2012 The Limits of the Self. Immunology and Biological Identity. New York: Oxford University Press.

Rosental, P.A. 2011 «Où s'arrête la contagion? Faits et utopie chez Gabriel Tarde», Tracés. Revue de sciences humaines 21(2): 109-124.

Serres, M. 1980 Le Parasite. Paris: Fayard.

Shapin, S. \& S. Schaffer 1993 Le Léviathan et la pompe à air. Hobbes et Boyle entre science et politique. Paris: La Découverte.

Tomes, N. 1998 The Gospel of Germs: Men, Women, and the Microbe in American Life. Cambridge: Harvard University Press.

Turnbaugh, P.J., Ley, R. E., Hamady, M., Fraser-Liggett, C.M., et al. 2007 «The human microbiome project», Nature 449: 804-810.

Worboys, M. 2001 «From heredity to infection : Tuberculosis, 1870-1890» in J.-P. Gaudillière, I. Lowy (dir.) Heredity and Infection: The History of Disease Transmission. Londres: Routledge.

Young, E. 2017 Moi microbiome maitre du monde. Préface P. Debré. Paris: Dunod.

Zylberman, P. 2013 Tempêtes microbiennes. Essai sur la politique de sécurité sanitaire dans le monde transatlantique. Paris: Gallimard.

\section{Pour citer cet article}

Keck, F. 2017 «Anthropologie des microbes. L'oubli de l'immunologie et la révolution du microbiome», Techniques E Culture 68 «Mondes infimes », p. 230-247. 